Relations industrielles

Industrial Relations

\title{
Lastra Lastra, José Manuel, Derecho sindical
}

\section{René Laperrière}

Volume 55, numéro 3, 2000

URI : https://id.erudit.org/iderudit/051345ar

DOI : https://doi.org/10.7202/051345ar

Aller au sommaire du numéro

\section{Éditeur(s)}

Département des relations industrielles de l'Université Laval

\section{ISSN}

0034-379X (imprimé)

1703-8138 (numérique)

Découvrir la revue

Citer ce compte rendu

Laperrière, R. (2000). Compte rendu de [Lastra Lastra, José Manuel, Derecho sindical]. Relations industrielles / Industrial Relations, 55(3), 557-559.

https://doi.org/10.7202/051345ar

Tous droits réservés ( Département des relations industrielles de l'Université Laval, 2000
Ce document est protégé par la loi sur le droit d'auteur. L'utilisation des services d'Érudit (y compris la reproduction) est assujettie à sa politique d'utilisation que vous pouvez consulter en ligne.

https://apropos.erudit.org/fr/usagers/politique-dutilisation/ 
and benefits enjoyed by their blue collar counterparts. When unionists fought for parity, they were thinking of parity between white and blue collar workers, not between men and women within their own union.

This book is well worth the attention of those interested in industrial relations, in women in the trade movement and in the history of what trade unions actually did following World War II. While making good use of personal interviews, Creese writes the kind of thoughtful and socially relevant historical analysis that is made possible when unions ensure that their records are preserved. Indeed, Creese recognizes that the one weakness of her study is her inability to get access to equally valuable management records. She has been able to get some sense of management's position on some of these issues, but this really is an analysis that focuses on union behaviour and activity. The broader corporate and social context within which the trade union negotiated is outlined and acknowledged when necessary, but it remains shadowy at best. The reader soon becomes aware of this limitation, but it does not detract from the overall effectiveness or quality of Creese's important book.

Ken Cruikshank McMaster University

\section{Derecho sindical}

par José Manuel Lastra Lastra, $3^{e}$ édition actualisée, México : Editorial Porrúa, 1999, 308 p., ISBN 970-07-1775-5.

L'ouvrage du professeur Lastra Lastra, professeur titulaire de droit du travail à l'Université nationale autonome du Mexique (UNAM), dépasse largement la description du droit positif mexicain pour proposer une approche historique et philosophique du droit du travail, examiné surtout dans ses rapports avec la liberté et la démocratie syndicales. Dans sa préface, le professeur Néstor de Buen souligne à juste titre l'ampleur de l'ouvrage, sa rigueur et sa pertinence actuelle. Nous ajouterons que l'auteur se préoccupe aussi de philosophie du droit et de droits fondamentaux, comme le montre un autre de ses ouvrages, Fundamentos de derecho, publié chez McGraw-Hill ( ${ }^{\mathrm{e}}$ éd., 1998), qui comprend notamment tout un chapitre (IX) consacré au travail dans la Constitution mexicaine (159-220).

Cette préoccupation constante pour les principes et les normes juridiques, pour la recherche de la justice, spécialement la justice sociale, nous paraît une des caractéristiques de la doctrine juridique latino-américaine. Elle se double chez Lastra Lastra d'une vaste culture à la fois juridique et laborale, si on veut bien nous permettre cet hispanisme. Jugeons-en plutôt par les sujets qu'il aborde dans ses six premiers chapitres, consacrés au travail dans l'histoire (de l'esclavage en Grèce jusqu'aux corporations médiévales), à la révolution industrielle et au mouvement ouvrier en Angleterre, à l'évolution des corporations de métiers en France, à l'organisation corporative en Italie, au syndicalisme espagnol et au mouvement ouvrier allemand. Ce grand panorama aide à comprendre la variété des structures syndicales, de leurs idéologies et de leurs pratiques; et s'il ne nous amène pas également pour tous les pays concernés jusqu'à l'aube du XXI $\mathrm{X}^{\mathrm{e}}$ siècle, c'est que l'auteur désire surtout montrer les évolutions générales qui ont façonné le syndicalisme et le droit de ces pays.

Ce tour d'horizon international se conclut par un chapitre traitant de la doctrine sociale de l'Église catholique romaine. Rares sont les ouvrages francophones de droit du travail qui se soient préoccupés de cette dimension, alors qu'en Amérique latine l'idéologie et la 
pratique religieuses sont encore bien vivantes. Depuis Rerum Novarum jusqu'à Centesimus Annus sont évoqués les enseignements de l'Église : ce compte rendu très synthétique est suivi d'une réflexion personnelle de l'auteur qui déplore qu'en cette fin de siècle les valeurs mises de l'avant par la doctrine sociale de l'Église aient été reléguées aux oubliettes pour céder la place à celles de compétitivité et de productivité, de flexibilisation et de déréglementation, avec la désaffection de l'État envers la protection sociale et le culte renouvelé d'un laissez-faire profitable au capital et aux entreprises. On voit ainsi l'utilité d'avoir dressé l'histoire du travail pour montrer à quel point le droit du travail est en danger de régression. Par contre, le caractère forcément synthétique de la présentation n'a pas permis à l'auteur d'élaborer sur la dénonciation générale que fait le Pape actuel des erreurs fondamentales du socialisme, qui d'un point de vue non catholique peut paraitre, sinon politiquement intéressée, du moins idéologiquement discutable.

La seconde partie de l'ouvrage traite plus spécifiquement du droit syndical mexicain : l'histoire du travail au Mexique, la consécration de la liberté syndicale dans la Constitution de 1917 et le droit d'association actuel. Suit une description des structures syndicales mexicaines, et un chapitre entier consacré à la liberté syndicale, question fondamentale très sensible et très discutée au Mexique. S'appuyant sur une grande variété de sources des pays à tradition romano-civiliste et sur la teneur du droit international, notamment la Constitution de l'OIT, l'auteur examine les fondements de la liberté syndicale et les principaux obstacles qui l'entravent au Mexique, notamment les clauses d'admission et d'exclusion syndicale (ateliers fermés) qui permettent aux dirigeants d'exercer une discipline serrée et ouvrent la porte à de graves abus.

L'égalité des chances et la discrimination dans l'emploi, sous toutes ses formes, font aussi l'objet d'importants développements, qui dépassent la question de la liberté syndicale au sens strict pour s'étendre aux diverses situations de travail, et qui dénotent le parti pris humaniste et égalitariste de l'auteur. Il rappelle que les traités ratifiés par le Mexique ont automatiquement force de loi au pays, selon les termes de la Constitution (ce qui ne correspond certes pas à la situation canadienne, tant s'en faut). Nous retiendrons comme particulièrement pertinentes les remarques de l'auteur sur la discrimination raciale, dans un pays où la population autochtone est importante et même emblématique de la « mexicanité ». L'affirmation de l'égalité est transposée au plan international, ce qui ne manque pas d'à propos compte tenu que le Mexique est à la fois un pays d'immigration (notamment de son voisin du sud, le Guatemala) et d'émigration (on estime à 400000 par année le nombre de Mexicains qui émigrent aux États-Unis d'Amérique). Quant à la discrimination sexuelle, l'auteur note surtout les disparités de revenus dans plusieurs pays, dont le Canada, qui persistent malgré certaines évolutions ; il déplore les difficultés socio-culturelles d'accès des femmes au marché du travail, et dénonce le harcèlement sexuel non seulement pour la quête de faveurs sexuelles de la part d'un supérieur (à laquelle se limite par exemple le Code du travail français) mais aussi pour le maintien d'un climat de travail empoisonné. Ouverture d'esprit qui contredit certains préjugés courants envers les cultures latines.

Le chapitre sur la structure et le fonctionnement des syndicats (statuts, personnalité juridique...), aborde la question très controversée du registre syndical, moyen de contrôle par excellence du pouvoir politique et judiciaire, et qui accorde au syndicat requérant les moyens juridiques d'agir (à l'instar de notre accréditation). Après avoir défini les principes et campé les positions, l'auteur va chercher son inspiration dans 
le droit constitutionnel comparé (Italie, Espagne, Allemagne, Russie, Pologne, Argentine, Colombie, Panama, Pérou...) pour constater à quel point ces procédures, destinées à légaliser l'action syndicale, peuvent au contraire être utilisées dans certains pays pour l'entraver ou l'étouffer dans l'œuf. Autre question délicate : la participation politique des syndicats. Elle est inévitable, selon l'auteur, et même désirable ; pourtant, elle doit s'opérer avec discernement et indépendance à l'égard des partis politiques, remarque pertinente dans un pays où les relations du travail sont façonnées par l'alliance historique entre le syndicalisme officiel et le parti au pouvoir. En ce qui touche enfin la concertation sociale, institution très développée au Mexique et fort discutée par ailleurs (à l'instar de nos « sommets économiques » québécois), elle fait l'objet d'une analyse serrée et l'auteur ne se prive pas de critiquer à la fois la supposée représentativité des organisations, la faible effectivité juridique des pactes sociaux qui ne comportent aucune obligation ferme, l'injustice de leurs résultats (puisque, comme chez nous, les mesures anti-inflationnistes n'ont effectivement contrôlé que les salaires, et non les prix ni les profits) et la trahison de leurs promesses (notamment par l'aggravation catastrophique du chômage et de l'inflation).

La fin de siècle paraît sombre à Lastra Lastra, qui termine tout de même sur une note d'espoir dans son dernier chapitre consacré aux perspectives actuelles du droit du travail. L'auteur tient à revaloriser le travail, dans un contexte économique qui cherche à l'exploiter ou à s'en défaire. Sa position humaniste le rapproche des penseurs idéalistes et de la doctrine de l'Église catholique qui voudraient maintenir la valeur du travail comme source de dignité humaine et comme moyen privilégié de subsistance et de participation sociale. Dans un tel processus, le droit fait cuvre de justification et d'institutionnalisation, sur la base de l'éthique et des principes de justice sociale. On est loin de certains courants européens qui cherchent à réduire considérablement le rôle du travail - et celui du droit - pour repenser la distribution des revenus et la participation sociale sous d'autres formes. Ici, l'auteur s'en prend particulièrement aux « Principes pour une nouvelle culture du travail $»$, objet d'un accord patronalsyndical de 1996 dont il dénonce l'enflure verbale et le manque de principes, alors que l'article 123 de la Constitution et la Loi fédérale du travail de 1970 énoncent déjà clairement les principes essentiels qui doivent régir le travail humain, notamment la stabilité et la continuité de l'emploi, l'égalité salariale, l'application de la norme la plus avantageuse et l'impossibilité de renoncer à ses droits, tout le contraire du modèle néo-libéral qu'on cherche à promouvoir par cet accord. L'auteur déplore enfin les effets négatifs de la mondialisation, qui fait du travail une valeur commerciale parmi d'autres, au détriment du plein emploi productif, un objet de concurrence entre les nations et un facteur d'aggravation de la précarité et de la pauvreté des populations.

En somme, la lecture de cet ouvrage mexicain est rafraîchissante, sans doute parce que, contrairement aux descriptions ou traductions en langue anglaise, ou plus rarement française, destinées à informer le lecteur sur l'état du droit positif (point de départ obligé de la démarche comparatiste), le présent livre nous montre l'ampleur de la culture juridique latino-américaine, nous fait pénétrer dans l'univers philosophique des juristes du travail, nous fait voir que les normes et institutions juridiques occupent une place et remplissent des fonctions différentes des nôtres. Nous pouvons ainsi mieux mesurer ce qui nous rapproche et ce qui nous distingue, et surtout constater que nous en avons beaucoup à apprendre des juristes mexicains et latino-américains.

RENÉ LAPERRIÈre

Université du Québec à Montréal 